\title{
Population-level sexual behaviours in adolescent girls before and after introduction of the human papillomavirus vaccine (2003-2013)
}

\author{
Gina S. Ogilvie MD DrPH, Felicia Phan MPH, Heather N. Pedersen MPH, Simon R. Dobson MD, \\ Monika Naus MD MHSc, Elizabeth M. Saewyc PhD RN
}

Cite as: CMAJ 2018 October 15;190:E1221-6. doi: 10.1503/cmaj.180628

Visual abstract available at www.cmaj.ca/lookup/suppl/doi:10.1503/cmaj.180628/-/DC1

\begin{abstract}
BACKGROUND: The human papillomavirus (HPV) vaccine is delivered widely through school-based immunization programs. Some groups have expressed concern that HPV vaccination programs will result in an increase in sexual risktaking behaviours among adolescents. We aimed to evaluate population-level changes in sexual behaviours before and after implementation of the schoolbased HPV vaccination program in British Columbia.
\end{abstract}

METHODS: In 2008, a school-based HPV vaccination program for girls was introduced in British Columbia. Using data from the BC Adolescent Health Survey - a longitudinal provincial survey administered in schools to capture adolescent physical and emotional health indicators, we conducted a linear trend analysis on sexual health behaviours and risk factors in adolescent girls before and after the implementation of vaccination for HPV (2003, 2008 and 2013).

RESULTS: We analyzed data for 298265 girls who self-identified as heterosexual. The proportion of girls reporting ever having sexual intercourse decreased from $21.3 \%$ (2003) to $18.3 \%$ (2013; adjusted odds ratio [OR] 0.79). Self-report of sexual intercourse before the age of 14 years decreased significantly from 2008 to 2013 (adjusted OR 0.76), as did reported substance use before intercourse (adjusted OR for 2003-2013 0.69). There was no significant change in the number of sexual partners reported (2003-2013). Between 2003 and 2013, girls' reported use of contraception and condoms increased, while pregnancy rates decreased.

INTERPRETATION: Since the implementation of school-based HPV vaccination program in $\mathrm{BC}$, sexual risk behaviours reported by adolescent girls either reduced or stayed the same. These findings contribute evidence against any association between HPV vaccination and risky sexual behaviours.
H

uman papillomavirus (HPV) is the most commonly diagnosed sexually transmitted infection in the world. ${ }^{1}$ While the majority of HPV infections are transient and resolve naturally without intervention, persistent infection with high-risk types of HPV can cause precancerous lesions that may progress to cervical, anal, oropharyngeal or other reproductive tract cancers, or anogenital warts if left untreated. ${ }^{2}$ There is evidence that vaccination programs for HPV effectively reduce rates of high-risk types of HPV at the population and clinical level.,4 Cervarix (GlaxoSmithKline), Gardasil and Gardasil-9 (Merck) are the 3 vaccines approved by Health Canada ${ }^{5}$ and recommended for use by the Canadian National Advisory Committee on Immunization. ${ }^{6}$ All 3 vaccines protect against HPV types 16 and 18, which are responsible for $70 \%$ of all cervical cancers. ${ }^{2}$ Gardasil and Gardasil-9 reduce the risk of additional types of HPV that are associated with genital warts and other cancers.

Many countries have implemented national publicly funded immunization programs for HPV. These programs are offered typically in school to adolescent girls aged 9-13 years and, in recent years, to boys. ${ }^{7}$ School-based programs for HPV vaccination have shown broad coverage among adolescent populations compared with other approaches. ${ }^{8}$ However, negative beliefs about HPV vaccination may present barriers to uptake. Parents of adolescent girls have expressed concern that HPV vaccination at a young age would encourage risky sexual behaviours such as early sexual debut, more sexual partners or unprotected intercourse, ${ }^{9-11}$ and that by consenting to having their child receive the HPV vaccine that they would be condoning sexual activity at younger ages. ${ }^{12}$ 
Some evidence from clinic-based populations, billings by physicians and cross-sectional studies of existing cohorts suggests no association between HPV vaccination and increased sexual risk behaviours after vaccination. ${ }^{13-15}$ However, few studies have examined population-level sexual behaviours over time. In this study, we used an established provincial adolescent health survey to evaluate population-level changes in sexual health and risky behaviours over a decade, before and after the implementation of the school-based HPV vaccination program, in British Columbia, Canada. ${ }^{11}$

\section{Methods}

\section{Survey design and methods}

The BC Adolescent Health Survey has been conducted every 5 or 6 years since 1992 and represents more than 1.4 million students enrolled in public schools in the province. ${ }^{16}$ It is a clusterstratified randomized survey of classrooms in grades 7 through 12 in public schools across BC, administered between January and June of the relevant year. Students complete the questionnaire (comprising about 140 items) by hand, facilitated by public health nurses and nursing students. ${ }^{16}$ Questions included those about demographic information, health, risky behaviours, health protective factors and respondents' experiences concerning health and other risk exposures. The survey is anonymous and does not capture identifiable data. Survey items were derived from existing surveys in Canada and the United States ${ }^{17}$ or pilottested based on community consultation and testing. ${ }^{16}$ Only items that were the same across all 3 survey years were included in this analysis. The overall participation rate in the survey is about $75 \%$ each year. Additional descriptions of survey methods have been published previously. ${ }^{16}$

\section{School program for human papillomavirus vaccination} In September 2008, BC launched a publicly funded, school-based routine program for HPV vaccination. ${ }^{18}$ During the first 3 years of the program, girls in grades 6 and 9 (born in 1994 to 1997) were offered vaccination for HPV. After 2011, the program was available for girls in grade 6 only, reaching those born in 1997 and later. Between 2008 and 2014, girls were offered a 3-dose vaccination schedule, which changed to a 2-dose schedule after 2014 for girls under the age of 15 years who were starting the series.

The school-based HPV vaccine program was not available for girls who completed the survey in 2003 and 2008. For 2013, all survey participants would have had the opportunity to receive HPV vaccination if they attended a BC school in grade 6 or 9 . We obtained uptake rates for HPV vaccination from the immunization coverage reports provided by the BC Centre for Disease Control, which capture all HPV vaccinations in the province. Coverage rates were defined as the proportion of female students enrolled in the relevant grade ( 6 or 9 ) in the specified year who were up to date with HPV vaccination by June $30 . .^{19,20}$

\section{Sampling}

We analyzed data from the 2003, 2008 and 2013 BC Adolescent Health Surveys. We stratified by grade and health service delivery area (there are 16 areas within the 5 regional health authorities in $\mathrm{BC}$ ) to obtain a representative sample at provincial and regional levels. The sample size was adjusted within each grade and health service delivery area stratum to ensure standard errors of $3.5 \%$ or lower. In school districts that required signed parental consent, the response rate was lower than in school districts that required parental notification and student consent. Therefore, we over-sampled these districts (about 25\%-30\% of participating school districts). We included only the school districts that had participated in all 3 survey rounds; 46 of the 59 school districts (77.9\%) met this criterion.

We weighted data after survey completion to adjust for differential probability of sampling and differential response rates and scaled to provincial enrolment by consultants at Statistics Canada. For this analysis, we included only adolescent girls who indicated that they were heterosexual, unsure, questioning or without attractions in the final weighted sample. Girls who identified as lesbian or bisexual (who represented $<5 \%$ of the total sample) will be examined in a separate analysis, because there may be differences in sexual decision-making among girls who identify as lesbian or bisexual and, therefore, it is unclear whether the $\mathrm{HPV}$ vaccine would have comparable effect.

\section{Measures}

Measures included demographic information (age, urban or rural residence and whether born in (anada) and selected sexual health behaviours (ever having sexual intercourse, age at first intercourse, number of sexual partners within the past year, substance use before last intercourse, use of condoms for last intercourse, use of birth control pills at last intercourse and pregnancy involvement). Additional descriptions of study measures and definitions have been published previously. ${ }^{16}$

\section{Statistical analysis}

We used SPSS Complex Samples version 22.0 (IBM) to adjust for complex cluster-stratified sampling and weighted data. We reported the prevalence of self-reported sexual behaviours among heterosexual girls for each year. To test the linear trend of sexual behaviours, contraception use and pregnancy involvement across years, we used age-adjusted logistic regression models (reference year 2013). Adjusted odds ratios (ORs) less than 1 indicated that the trend (or odds) of the given sexual behaviour in the earlier survey year was greater than in 2013, and adjusted ORs greater than 1 indicated that the odds of the behaviour in the earlier survey were less than in 2013.

\section{Ethics approval}

The study was approved by the Behavioural Research Ethics Board at The University of British Columbia (H12-02630).

\section{Results}

The weighted and scaled population estimate for adolescent girls who identified as heterosexual or were unsure was 302626 (Table 1), of whom 123166 (40.7\%) participated in 2003, 99925 (33.0\%) in 2008 (prevaccination group) and 79535 
Table 1: Characteristics of female participants in the BC Adolescent Health Survey, by years 2003, 2008 and 2013

\begin{tabular}{|c|c|c|c|}
\hline \multirow[b]{2}{*}{ Characteristic } & \multicolumn{3}{|c|}{$\begin{array}{l}\%(95 \% \mathrm{Cl}) \text { of participants who identified as heterosexual* } \\
\qquad n=302626\end{array}$} \\
\hline & $2003 \dagger$ & $2008 \dagger$ & 2013ł \\
\hline Total population weighted estimate,§ $n$ (\% ) & $123166(40.7)$ & $99925(33.0)$ & 79535 (26.3) \\
\hline Age, mean $\pm S D ; y r$ & $14.87(14.80-14.93)$ & $14.98(14.94-15.03)$ & $14.89(14.85-14.93)$ \\
\hline School grade, mean \pm SD & $9.45(9.39-9.51)$ & $9.66(9.60-9.73)$ & $9.62(9.58-9.66)$ \\
\hline \multicolumn{4}{|l|}{ Residence area } \\
\hline Urban & $86.9(85.6-88.1)$ & $86.6(85.0-88.1)$ & $88.0(86.3-89.6)$ \\
\hline Rural & $13.1(11.9-14.4)$ & $13.4(11.9-15.0)$ & $12.0(10.4-13.7)$ \\
\hline \multicolumn{4}{|l|}{ Born in Canada } \\
\hline Yes & $82.0(80.1-83.7)$ & $81.5(80.3-82.6)$ & $81.3(80.1-82.4)$ \\
\hline No & $18.0(16.3-19.9)$ & $18.5(17.4-19.7)$ & $18.7(17.6-19.9)$ \\
\hline \multicolumn{4}{|c|}{$\begin{array}{l}\text { Note: } \mathrm{Cl}=\text { confidence interval, } \mathrm{HPV}=\text { human papillomavirus, } \mathrm{SD}=\text { standard deviation. } \\
\text { *Unless stated otherwise. } \\
\text { tSurvey years before implementation of the school-based HPV vaccination program in British Columbia. } \\
\text { ISurvey year after implementation of the school-based HPV vaccination program in BC. All girls surveyed would have had the opportunity to receive the } \\
\text { HPV vaccine. } \\
\text { \$Weighted and scaled sample represents this population of adolescent girls in BC who were heterosexual. }\end{array}$} \\
\hline
\end{tabular}

(26.3\%) in 2013 (postvaccination group). Demographic factors are presented in Table 1 . The age range was 12-18 years for girls in all years. Most girls lived in urban areas (between $86.6 \%$ and $88.0 \%$ for all years), and most were born in Canada ( $81.3 \%-82 \%$ for all years). In the first year of the BC schoolbased HPV vaccination program (2008/09), uptake rates were $61.8 \%$ among female students in grade 6 . Rates decreased slightly the following year but then increased over time to $68.8 \%$ in $2013 .{ }^{19}$ Among female students in grade $9,62.1 \%$ of eligible girls were vaccinated in 2008/09, and rates decreased slightly to $61.7 \%$ in 2011 (Table 2). ${ }^{20}$

Participants were asked whether they had had sex in their lifetime, and only those with valid responses were included in the analysis (population estimate 298265; $1.5 \%$ of responses were missing from the demographic population). Only girls who responded yes to this question answered subsequent questions related to sexual behaviours. Prevalence and adjusted OR between time points among girls who identified as heterosexual are presented in Table 3. The prevalence of girls reporting ever having sexual intercourse decreased steadily between survey years, from $21.3 \%$ in 2003 to $20.6 \%$ in 2008 to $18.3 \%$ in 2013 . When comparing prevaccination years (2003 and 2008) with the postvaccination year (2013), the adjusted ORs for ever having sexual intercourse were 0.79 (95\% confidence interval [Cl] $0.71-0.88)$ and $0.89(95 \% \mathrm{Cl} 0.82-$ $0.98)$, respectively, which indicates that girls were significantly less likely to report ever having sex after introduction of the HPV vaccination program.

Among those who have ever had intercourse, the proportion having intercourse before the age of 14 years also decreased over time, with a significant change observed between 2008 and 2013 (adjusted OR 0.76, 95\% Cl 0.61-0.96). There was no significant change in the prevalence of having 3 or more sexual partners over time (adjusted OR for 2003-2013 1.02, 95\% Cl 0.83-1.26; adjusted OR for 2008-2013 0.90, 95\% Cl 0.75-1.09). The percentage of girls who used substances such as alcohol or drugs before their last intercourse increased slightly between $2003(26.0 \%)$ and 2008 (28.3\%), and then decreased in 2013 $(19.3 \%)$. The trend analyses showed that prevalence of substance use before last intercourse decreased after implementation of the HPV vaccination program (adjusted OR for 2003-2013 0.69, $95 \% \mathrm{Cl}$ 0.58-0.82).

Reported use of condoms and birth control pills increased from previous years to 2013. Condom use was $65.6 \%$ in 2003, which decreased to $63.3 \%$ in 2008 and increased to $68.9 \%$ in 2013. Condom use significantly increased from previous years to 2013 after implementation of the HPV vaccination program (adjusted OR for 2003-2013 1.19, 95\% Cl 1.02-1.39). Oral contraceptive use

Table 2: Coverage rates of the school-based vaccination program for human papillomavirus among female participants in grades 6 and 9 in British Columbia, 2008-2013

$\%$ of participants who completed the series (by year of grade completion)

\begin{tabular}{lccccc|}
$\begin{array}{l}\text { Grade in } \\
\text { school }\end{array}$ & $\mathbf{2 0 0 9}$ & $\mathbf{2 0 1 0}$ & $\mathbf{2 0 1 1}$ & $\mathbf{2 0 1 2}$ & $\mathbf{2 0 1 3}$ \\
\hline 6 & 61.8 & 59.9 & 68.2 & 68.7 & 68.8 \\
\hline 9 & 62.1 & 58.1 & 61.7 & $*$ & * \\
\hline
\end{tabular}

Note: $\mathrm{HPV}=$ human papillomavirus.

${ }^{*}$ Coverage rates for girls who were in grade 9 in 2011/12 and 2012/13 were not assessed because these girls had been offered 3 doses of the HPV vaccine in grade 6 in $2008 / 09$ and 2009/10, respectively. Therefore, their coverage rates can be observed in the grade 6 results. 
Table 3: Sexual behaviours in female participants between 2003 and 2013, based on the BC Adolescent Health Survey

$\%(95 \% \mathrm{CI})$ of participants*

Total population weighted estimate§, $n$

$2003 \dagger$

$2008 \dagger$

98588

$2013 \ddagger$

78322
Behaviour

Age-adjusted OR (95\% CI)

Trend 2003-2013

Trend 2008-2013

Sexual risk behaviour

Ever had sexual intercourse

Early first intercourse (before age $14 \mathrm{yr}$ )

Three or more sexual partners within the past year

Used substances before last intercourse

\section{Contraceptive use}

Used condoms at last intercourse

Used birth control pills at last intercourse

\section{Pregnancy involvement}

Ever been pregnant

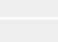

\begin{tabular}{|c|c|c|c|c|}
\hline $21.3(20.0-22.5)$ & $20.6(19.7-21.5)$ & $18.3(17.4-19.3)$ & $0.79(0.71-0.88)$ & $0.89(0.82-0.98)$ \\
\hline $14.3(12.7-16.2)$ & $13.0(11.6-14.7)$ & $10.2(8.7-11.8)$ & $0.82(0.65-1.03)$ & $0.76(0.61-0.96)$ \\
\hline $15.5(13.4-17.8)$ & $17.1(15.4-18.9)$ & $15.7(14.0-17.6)$ & $1.02(0.83-1.26)$ & $0.90(0.75-1.09)$ \\
\hline $26.0(23.7-28.5)$ & $28.3(26.2-30.6)$ & $19.3(17.4-21.3)$ & $0.69(0.58-0.82)$ & $0.60(0.51-0.71)$ \\
\hline $65.6(63.2-68.1)$ & $63.3(60.9-65.7)$ & $68.9(66.4-71.3)$ & $1.19(1.02-1.39)$ & $1.28(1.10-1.49)$ \\
\hline $45.1(42.2-48.0)$ & $48.1(45.7-50.5)$ & $54.5(52.0-57.0)$ & $1.43(1.21-1.67)$ & $1.26(1.10-1.45)$ \\
\hline $5.9(4.6-7.3)$ & $5.0(4.1-6.1)$ & $3.4(2.6-4.5)$ & $0.56(0.40-0.80)$ & $0.69(0.49-0.98)$ \\
\hline
\end{tabular}

t

Note: $\mathrm{Cl}=$ confidence interval, $\mathrm{HPV}=$ human papillomavirus, $\mathrm{OR}=$ odds ratio.

*Unless specified otherwise.

$\ddagger$ Survey year after the implementation of the school-based HPV vaccination program in BC. All girls surveyed would have had the opportunity to receive
§Weighted and scaled sample represents this population of adolescent heterosexual girls in BC who responded to the question on sexual intercourse. increased by 9.4\% between 2003 and 2013 (adjusted OR 1.43, $95 \% \mathrm{Cl} 1.21-1.67)$. The prevalence of girls who reported pregnancy decreased by 42\% from 2003 to 2013 (adjusted OR for 2003-2013 0.56, 95\% Cl 0.40-0.80).

\section{Interpretation}

After implementation of the publicly funded school-based vaccination program for HPV in September 2008 in BC, sexual behaviours and sexual health indicators among adolescent girls either improved toward safer sexual health practices or did not change significantly. Our analysis of data from the BC Adolescent Health Survey suggests that the implementation of a school-based program for HPV vaccination was not associated with an increase in risky sexual behaviours among adolescent girls at the population level.

Concerns that the HPV vaccine could lead to increasingly risky sexual behaviours originate from the perception that by participating in an intervention that reduces risk, one may engage in higher-risk behaviours (also called risk compensation $^{21}$ or risk homeostasis). ${ }^{22}$ Studies on risk perception after HPV vaccination suggest that related adolescent beliefs are not aligned with risk compensation theory. Two 2012 studies reported that, although adolescent girls who had received the HPV vaccine perceived themselves to be at lower risk of acquiring HPV, they did not perceive a lower risk for other sexually transmitted infections, and most reported the continued need for safer sexual practices. ${ }^{23,24}$ It is worth noting that a comprehensive vaccine education program was paired with the implementation of the HPV vaccination program in $\mathrm{BC} .{ }^{25}$ This could have provided participants with a better understanding of the limits of the HPV vaccine for protection against other sexually transmitted infections.

A prospective cohort study that used clinical indicators to measure sexual behaviours among 11- to 12-year-old girls enrolled in a managed care organization in the US, who were exposed and not exposed to the HPV vaccine, found no differences between groups in incidence of pregnancy, testing for sexually transmitted diseases or contraceptive counselling. ${ }^{15}$ Another study found no increased risk in composite outcomes of pregnancy and sexually transmitted infection among adolescent girls after implementation of Ontario's grade $8 \mathrm{HPV}$ vaccination program. ${ }^{13}$ These studies examined clinical outcomes related to risky sexual behaviours as a proxy for direct measures of such behaviours, such as age at first intercourse or number of sexual partners. Studies of self-reported direct measures of risky behaviours similarly reported no association between measures of sexual debut and HPV vaccination. ${ }^{26,27}$ Other research has also found no relationship between HPV vaccination and the number of sexual partners, ${ }^{26,27}$ which is consistent with the results of our study. In other research, use of condoms and contraceptives was consistently higher among young women who had received the vaccine than those who had not. ${ }^{4,26,27}$ We did not find other studies that examined the association between HPV vaccination and substance use before intercourse.

The main strength of our study is that it is representative of the $B C$ population because of its large sample size and complex sampling technique using an established provincial adolescent health survey, accompanied by a high response 
rate. Previous research has focused mainly on clinic-based populations, retrospective cohorts and smaller cross-sectional study samples.

\section{Limitations}

Our analysis has some limitations. We did not examine the direct relationship between individual HPV vaccination status and sexual behaviours, and we cannot infer individual-level or causal relationships because the Adolescent Health Survey does not capture identifiable data that can be linked to public health vaccination records. However, a self-reported question on HPV vaccination status is being included for future survey cycles. For this ecological study, we used time as an indicator to separate the prevaccination program population (2003 and 2008) from the postvaccination population (2013). We do not suggest that the observed sexual behaviours over time are attributable to the implementation of HPV vaccination in BC. Previous evidence from the survey has shown a downward trend in risky sexual behaviours since before 2003. ${ }^{16}$ Sexual and reproductive health behaviours are influenced by a complex interplay of contextual factors including the legal and political environment, as well as sociocultural and economic factors. ${ }^{28}$ There is evidence of a global trend toward safer sex practices since before the HPV vaccine was available..$^{29,30}$ In sub-Saharan Africa where HPV vaccination is mostly absent, a multicountry study using data from 2000 to 2010 observed trends toward older age of sexual debut and increasing use of contraception over time. ${ }^{31}$ Population data in the US for 1976-2016 showed that fewer adolescents have been engaging in behaviours such as dating, alcohol consumption and sex, with a sharp decline since 2000. ${ }^{30}$ Sexual behaviour data are self-reported and may be subject to recall and social desirability bias. However, this bias was likely present in all survey years.

\section{Conclusion}

Our finding that self-reported risky sexual behaviours reduced or stayed the same among adolescent girls in BC after introduction of a school HPV vaccination program with uptake of about $68 \%$ can help mitigate concerns based on risk compensation theory.

Future analyses should explore differences between girls who identify with other sexual orientations such as homosexuality and evaluate sexual behaviour among girls who received the HPV vaccine and those who did not. Additional studies could be conducted to explore national trends or examine differences between different provincial vaccination programs.

\section{References}

1. Baseman JG, Koutsky LA. The epidemiology of human papillomavirus infections. J Clin Virol 2005;32(Suppl 1):S16-24.

2. Stanley M. Pathology and epidemiology of HPV infection in females. Gynecol Oncol 2010;117(Suppl):S5-10.

3. Drolet M, Bénard É, Boily MC, et al. Population-level impact and herd effects following human papillomavirus vaccination programmes: a systematic review and meta-analysis. Lancet Infect Dis 2015;15:565-80.
4. Cummings T, Zimet GD, Brown D, et al. Reduction of HPV infections through vaccination among at-risk urban adolescents. Vaccine 2012;30:5496-9.

5. Drug Product Database: access the database. Ottawa: Health Canada; (modified 2015 June 18). Available: www.canada.ca/en/health-canada/services/ drugs-health-products/drug-products/drug-product-database.html (accessed 2018 Feb. 26).

6. Updated recommendations on human papillomavirus vaccines: 9-valent HPV vaccine and clarification of minimum intervals between doses in the HPV immunization schedule: an Advisory Committee Statement (ACS) National Advisory Committee on Immunization (NACI). Ottawa: Public Health Agency of Canada; 2017.

7. Bruni L, Diaz M, Barrionuevo-Rosas L, et al. Global estimates of human papillomavirus vaccination coverage by region and income level: a pooled analysis. Lancet Glob Health 2016;4:e453-63.

8. Paul P, Fabio A. Literature review of HPV vaccine delivery strategies: considerations for school- and non-school based immunization program. Vaccine 2014; 32:320-6.

9. Abdelmutti N, Hoffman-Goetz L. Risk messages about HPV, cervical cancer and the HPV vaccine gardasil in North American news magazines. $J$ Cancer Educ 2010;25:451-6.

10. Zimet GD, Rosberger Z, Fisher WA, et al. Beliefs, behaviors and HPV vaccine: correcting the myths and the misinformation. Prev Med 2013;57:414-8.

11. Ogilvie GS, Remple VP, Marra F, et al. Parental intention to have daughters receive the human papillomavirus vaccine. CMAJ 2007;177:1506-12.

12. Hendry M, Lewis R, Clements A, et al. "HPV? Never heard of it!": a systematic review of girls' and parents' information needs, views and preferences about human papillomavirus vaccination. Vaccine 2013;31:5152-67.

13. Smith LM, Kaufman JS, Strumpf EC, et al. Effect of human papillomavirus (HPV) vaccination on clinical indicators of sexual behaviour among adolescent girls: the Ontario Grade 8 HPV Vaccine Cohort Study. CMAJ 2015;187:E74-81.

14. Madhivanan P, Pierre-Victor D, Mukherjee S, et al. Human papillomavirus vaccination and sexual disinhibition in females: a systematic review. Am J Prev Med 2016;51:373-83.

15. Bednarczyk RA, Davis R, Ault K, et al. Sexual activity-related outcomes after human papillomavirus vaccination of 11- to 12-year-olds. Pediatrics 2012; 130:798-805.

16. Saewyc EM, Taylor D, Homma Y, et al. Trends in sexual health and risk behaviours among adolescent students in British Columbia. Can J Hum Sex 2008;17:1-13.

17. Add Health: The National Longitudinal Study of Adolescent to Adult Health Chapel Hill (NC): Carolina Population Center; 2018. Available: www.cpc.unc. edu/projects/addhealth (accessed 2018 Jan. 30).

18. Naus M, Ogilvie G. Human papillomavirus vaccine program in BC: a good start with room for improvement. B C Med J 2010;52:95.

19. Immunization uptake in Grade 6 students: 2002-2017. Vancouver: BC Centre for Disease Control; 2017. Available: www.bccdc.ca/resource-gallery/Documents/ Statistics\%20and\%20Research/Statistics\%20and\%20Reports/Immunization/ Coverage/Grade6_Coverage_Results_2017.pdf (accessed 2018 Jan. 26).

20. Immunization uptake in Grade 9 students: 2002-2017. Vancouver: BC Centre for Disease Control; 2017. Available: www.bccdc.ca/resource-gallery/Documents/ Statistics\%20and\%20Research/Statistics\%20and\%20Reports/Immunization/ Coverage/Grade9_Coverage_Results_2017.pdf (accessed 2018 Jan. 26).

21. Hogben M, Liddon N. Disinhibition and risk compensation: scope, definitions, and perspective. Sex Transm Dis 2008;35:1009-10.

22. Wilde GJ. Risk homeostasis theory: an overview. Inj Prev 1998;4:89-91.

23. Mullins TL, Zimet GD, Rosenthal SL, et al. Adolescent perceptions of risk and need for safer sexual behaviors after first human papillomavirus vaccination. Arch Pediatr Adolesc Med 2012;166:82-8.

24. Kahn JA, Xu J, Zimet GD, et al.; Adolescent Trials Network for HIV/AIDS Interventions. Risk perceptions after human papillomavirus vaccination in HIV-infected adolescents and young adult women. J Adolesc Health 2012;50:464-70.

25. Ogilvie G, Anderson M, Marra F, et al. A population-based evaluation of a publicly funded, school-based HPV vaccine program in British Columbia, Canada: parental factors associated with HPV vaccine receipt. PLoS Med 2010;7:e1000270. 
26. Hansen BT, Kjær SK, Arnheim-Dahlström L, et al. Human papillomavirus (HPV) vaccination and subsequent sexual behaviour: evidence from a large survey of Nordic women. Vaccine 2014;32:4945-53.

27. Liddon NC, Leichliter JS, Markowitz LE. Human papillomavirus vaccine and sexual behavior among adolescent and young women. Am J Prev Med 2012; 42:44-52.

28. Patton GC, Sawyer SM, Santelli JS, et al. Our future: a Lancet commission on adolescent health and wellbeing. Lancet 2016;387:2423-78.
29. Wellings K, Collumbien M, Slaymaker E, et al. Sexual behaviour in context: a global perspective. Lancet 2006;368:1706-28.

30. Twenge JM, Park H. The decline in adult activities among U.S. adolescents, 1976-2016. Child Dev 2017 Sept. 18 [Epub ahead of print]. doi: 10.1111/ cdev. 12930

31. Doyle AM, Mavedzenge SN, Plummer ML, et al. The sexual behaviour of adolescents in sub-Saharan Africa: patterns and trends from national surveys. Trop Med Int Health 2012;17:796-807.
Competing interests: Gina Ogilvie and Simon Dobson reported antibody assay testing received from Merck that was conducted as part of an independent study monitoring antibody response to the HPV vaccine. The assay testing was done at no cost to the study. No other competing interests were declared.

This article has been peer reviewed.

Affiliations: School of Population and Public Health (Ogilvie, Phan, Pedersen), Faculty of Medicine; School of Nursing (Saewyc), The University of British Columbia; Women's Health Research Institute (Ogilvie, Phan, Pedersen), BC Women's Hospital; Vaccine Evaluation Center (Dobson), BC Children's Hospital Research Centre; BC Centre for Disease Control (Naus), Vancouver, BC

Contributors: Gina Ogilvie, Simon Dobson, Monika Naus and Elizabeth Saewyc contributed substantially to the conception and design of the study. Monika Naus and Elizabeth Saewyc contributed substantially to data acquisition.
Felicia Phan and Elizabeth Saewyc contributed substantially to data analysis. Gina Ogilvie, Felicia Phan and Heather Pedersen substantially contributed to the interpretation of the data. Felicia Phan and Heather Pedersen drafted the initial manuscript. All of the authors reviewed and revised the manuscript critically for important intellectual content, gave final approval of the version to be published, and agreed to be accountable for all aspects of the work in ensuring that questions related to the accuracy or integrity of any part of the work are appropriately investigated and resolved.

Funding: This study was funded in part by Canadian Institutes of Health Research (ClHR) grant no. MOP 119472 to Elizabeth Saewyc (principal investigator) and a ClHR Foundation Grant to Gina Ogilvie (principal investigator).

Data sharing: The BC Adolescent Health Survey is conducted by the McCreary Centre Society; they give approval for data access to established health and social science researchers whose proposed research aligns with the original purposes of the survey. Data are available for analysis only on site at the McCreary Centre Society in Vancouver, BC, on its secure computers, after approval of the proposed analyses, a signed Memorandum of Understanding and payment of data access fees to provide computer access and technical support. Further information on accessing the BC Adolescent Health Surveys is available at www.mcs.bc.ca/research_partnerships.

Acknowledgements: The authors would like to thank Dr. Yuko Homma and Dr. Gu Li (Stigma and Resilience Among Vulnerable Youth Centre, The University of British Columbia), who provided some of the statistical analysis for the article.

Disclaimer: The funders had no role in study design, data collection and analysis, decision to publish or preparation of the manuscript.

Accepted: Aug. 20, 2018

Correspondence to: Gina Ogilvie, gina.ogilvie@cw.bc.ca 